\title{
Anti-phospholipid antibodies in women presenting with preterm delivery because of preeclampsia or placental insufficiency
}

\author{
(D) Rubina Izhar1, (D) Syed Hasan Ala², (D) Samia Husain ${ }^{1}$, (D) Sonia Husain ${ }^{1}$ \\ ${ }^{1}$ Department of Obstetrics and Gynecology, Karachi Medical and Dental College, Abbasi Shaheed Hospital, Karachi, Pakistan \\ 2Department of Obstetrics and Gynecology, Dow University of Health Sciences, Karachi, Pakistan
}

\section{Abstract}

Objective: To assess the proportion of women presenting with preterm delivery because of preeclampsia or placental insufficiency (PREPI) with anti-phospholipid antibodies (APLA).

Material and Methods: This was a prospective cohort study conducted at an obstetrics and gynecology department. Women, aged 20-40 years, with preeclampsia who delivered before 34 weeks were cases while those who delivered before 34 weeks but did not have preeclampsia acted as controls. Both groups had APLA measured at diagnosis and 12-weeks postnatally. Anti-phospholipid antibody syndrome (APS) was diagnosed according to Sapporo's criteria.

Results: The study included 98 cases and 106 controls. Both cases and controls were similar in terms of age, gestational age and parity. The frequency of APS positivity was $17.3 \%$ in cases but only $3.8 \%$ in controls $(p=0.001)$. Cases were more likely to be of Baloch ethnicity ( $34.7 \%$ vs. $11.3 \%, p=0.001)$, have a history of miscarriage ( $25.5 \%$ vs. $13.2 \%, p=0.026)$, use aspirin $(p<0.001)$ or low molecular weight heparin $(p<0.001)$, and be obese $(\mathrm{p}<0.001)$ than controls. Cases were more likely to have lupus anticoagulant antibodies $(82.4 \%$ vs. $75 \%)$.

Conclusion: Our study confirms a high prevalence of APLA in women who have preterm delivery due to PREPI. An opportunity to screen these women should be made, so that proper counselling can be given and future pregnancies can be managed in an appropriate and timely manner. (J Turk Ger Gynecol Assoc 2021; 22: 85-90)

Keywords: Anti-phospholipid antibody syndrome, preterm delivery, preeclampsia, Sapporo's criteria, screening

Received: 12 August, 2020 Accepted: 16 February, 2021

\section{Introduction}

Anti-phospholipid antibody syndrome (APS) is an autoimmune disorder associated with the presence of autoantibodies specific for a number of different phospholipid molecules including lupus anticoagulant (LAC), anti-cardiolipin antibodies (ACA) and anti- $\beta 2$-glycoprotein I (aß2GPI) antibodies. APS presents as a spectrum of clinical phenotypes, such as thrombosis in the veins, arteries and microvasculature or the sequelae of these thromboses, as well as obstetrical complications (1).

Thrombosis is the hallmark of APS and leads to complications such as recurrent miscarriage and fetal demise in early pregnancy. Late complications comprise pre-eclampsia, preterm delivery before 34 weeks and intrauterine growth restriction due to placental insufficiency.

Preterm delivery for preeclampsia or placental insufficiency (PREPI) is a clinical criterion for APS (2). Preeclampsia leads to high maternal mortality and morbidity and is also implicated in fetal and neonatal morbidity. Worldwide estimates range from 5-10 percent (3). Nulliparity, a family or personal history of preeclampsia, raised body mass index (BMI), advanced maternal age, chronic kidney disease, chronic hypertension and autoimmune disease are known risk factors. However, many risk factors are still under-researched (4). An association between anti-phospholipid antibodies (APLA) and preterm delivery due to placental disease has been proven by several 
case control and cohort studies. The majority of earlier studies have had small sample sizes, used variable definitions of preeclampsia and have frequently included women who develop preeclampsia at term (5-8). Some studies have included low levels of APLA titers, which are not in line with Sapporo's criteria. Therefore the evidence base for this association is not robust and there is a lack of quality studies that prove an association of APLA with pregnancy morbidity (9).

Only one prospective study has addressed this issue to date. However the authors mentioned that the study had a few limitations including that repeat testing was not performed in around half of the women due to a high drop out rate and thus the true proportion with APLA may be higher than that reported. Moreover, the study included women who had delivered before 36 weeks which is outside the specified limit employed by Sapporo's criteria, which is 34 weeks. This would have led to underestimation of the problem by including all women delivering up to 36 weeks as the denominator (10).

Screening for APLA is not widely employed due to the lack of robust evidence, clear consensus and cost constraints (11). Reliable data on the prevalence of APLA in women with PREPI would provide a rationale for establishing a screening policy for these antibodies in such women.

The aim of this study was to prospectively determine the proportion of women with preterm delivery before 34 weeks due to PREPI who also test positive for APLA.

\section{Material and Methods}

This was a prospective cohort study, conducted at a department of obstetrics and gynecology, from January 2019 to December 2019. Women aged 20-40 years with preeclampsia who delivered before 34 completed weeks were included as cases. For the study period, both preeclampsia and preeclampsia with severe features was defined as per the American College of Obstetricians and Gynecologists (ACOG) criteria (12). Women who delivered before 34 completed weeks but did not have preeclampsia were included as controls. Women who did not consent, had cervical incompetence, had premature prelabor rupture of membranes, had previous history of cervical trauma, cone biopsy, cervical surgery and women who had known connective tissue disorder were excluded. Patients with a history of chronic hypertension, a history of renal disease or with a history of diabetes mellitus affecting kidneys were also excluded.

After obtaining informed and written consent those with preeclampsia and the controls had APLA measured after delivery. Women were diagnosed with preeclampsia if they had systolic blood pressure greater than $140 \mathrm{mmHg}$ and/or diastolic blood pressure exceeded $90 \mathrm{mmHg}$. Furthermore, women with acute onset of high blood pressure, with or without proteinuria, along with one or more severe features were included.

For the purpose of the study, placental insufficiency was defined according to the international consensus for the diagnosis of APS (13). Placental insufficiency was defined as delivery due to intrauterine growth restriction, where the estimated weight of fetus was $<10^{\text {th }}$ percentile or abdominal circumference was found to be $<5^{\text {th }}$ percentile for gestational age. The fetus should have decreased liquor, defined as amniotic fluid index $<5 \mathrm{~cm}$ or deepest vertical pocket $<2 \mathrm{~cm}$, abnormal Doppler ultrasound suggestive of fetal hypoxemia (absent or reversed end diastolic flow), abnormal cardiotocography, or biophysical profile score $<6$.

For estimation of serum APLA levels, all blood samples were drawn by venipuncture in serum separator tubes. Blood samples were taken for APLA postnatally, and if the first test was positive, repeated 12 weeks later. Serum antibody levels were measured at a reference lab.

Women were diagnosed with APS according to Sapporo's criteria. Sapporo's criteria (13), classify a patient as true APS when at least one of the two clinical criteria are present and at least one of the two laboratory criteria are positive. Clinical criteria include the presence of vascular thrombosis or pregnancy associated morbidity, and laboratory criteria include the presence of medium or high titers of immunoglobulin $\mathrm{G}$ (IgG) or IgM ACA or a $\beta 2$ GPI antibodies by enzyme-linked immunosorbent assay methods or the presence of LAC, based on International Society on Thrombosis and Hemostasis criteria, measured on two or more occasions at least 12 weeks apart (14).

The primary outcome measure was presence or absence of APLA which was defined as any single positive antiphospholipid test, including LAC, ACA IgG (GPL) or IgM (MPL) >40, and a $\beta 2$ GPI IgG (SGU) or IgM (SMU) > 40 (14). As an individual may test positive for more than one antibody at the same time, each antibody was tested separately in all participants.

\section{Statistical analysis}

It is reported that women with PREPI have increased odds of having APLA compared to controls (11.5\% vs. 1.4\%, respectively) (10) With a power of $80 \%(1-\beta)$ and a one-sided 0.05 risk of type 1 error $(\alpha)$, the sample size would need to be 93 patients with preterm delivery in each group to demonstrate a greater proportion of women with PREPI would have APLA. In order to compensate for protocol deviation the sample size was inflated $5 \%$ and a minimum of 100 women were to be included in each arm.

Data was analyzed using SPSS, version 16 (IBM Inc., Armonk, NY, USA). Shapiro-Wilk's test was used to assess normality of data distribution. Mean and standard deviations were calculated 
for the quantitative variables, such as maternal age, BMI, and parity. Frequencies and percentages were calculated for the qualitative variables, including ethnicity, history of miscarriage, history of aspirin use, low molecular weight heparin use, obesity (BMI $>30 \mathrm{~kg} / \mathrm{m}^{2}$ ), mode of delivery, presence of APS, and LAC and ACA, a $\beta 2$ GPI antibodies. Effect modifiers were controlled through stratification of maternal age, BMI, parity, ethnicity, history of miscarriage, history of aspirin or low molecular weight heparin use, and obesity, to investigate the effect of these on the outcome variable. Post stratification chi square test was applied taking a p-value of 0.05 as statistically significant.

The study was approved by the Institutional Ethics Committee of Aziz Medical Center (approval number: IEC/AZIZ/334). All participants provided informed consent.

\section{Results}

During the study 230 women satisfied the inclusion criteria and were approached for the study. Of these, 12 women refused to participate. Of the remaining 218, 103 (47.25\%) women delivered preterm due to PREPI and 115 (52.75\%) delivered preterm without preeclampsia. Of those with PREPI, four women were lost to follow up and one refused to undergo the second test for APA and these were excluded. Of the controls, seven women were lost to follow up and two who did not get tested at the reference lab were excluded.

We therefore included 98 cases and 106 controls. The basic characteristics of these women are summarized in Table 1. The mean age of participants was $30.98 \pm 3.92$ years and had a mean gestational age at delivery of $33.19 \pm 1.80$ weeks. Most (97, 47.5\%) had two children, the largest ethnicity was Sindhi (31.9\%) and most delivered vaginally (78.4\%). A total of 39 (19.1\%) had a history of miscarriage and 41 (20.1\%) were obese. There was a history of aspirin use in 49 (24\%) women, while $11.7 \%$ used low molecular weight heparin. Both cases and controls were similar in terms of age, gestational age in weeks and parity.

Cases were more likely to be Baloch ethnicity $(34.7 \%$ vs. $11.3 \%, \mathrm{p}=0.001)$, have a history of miscarriage $(25.5 \%$ vs. $13.2 \%, \mathrm{p}=0.026)$ and were using aspirin $(\mathrm{p}<0.001)$, low molecular weight heparin $(\mathrm{p}<0.001)$, and were more obese $(\mathrm{p}<0.001)$ than controls (Table 2$)$. Among the study population, significantly more cases $(n=17,17.3 \%)$ had APLA compared with controls $(n=4,3.8 \% ; p=0.001)$.

ACA were present in $47.1 \%$ (8/17) of cases and 50\% (2/4) of controls, who tested positive for APA, while LAC was positive in $82.4 \%(14 / 17)$ of cases and $75 \%(3 / 4)$ of controls. A $\beta 2 \mathrm{GPI}$ was positive in $17.6 \%$ (3/17) of cases and $25 \%$ (1/4) of controls (Table 3).

\section{Discussion}

\section{Main findings}

The present study assessed the proportion of women with PREPI who had APLA. Our study shows that women with PREPI are more likely to have APLA compared with those who do not have PREPI.

Previous history of miscarriage, aspirin and low molecular weight heparin use and obesity were common in women with PREPI who deliver preterm.

Table 1. Characteristics of study population

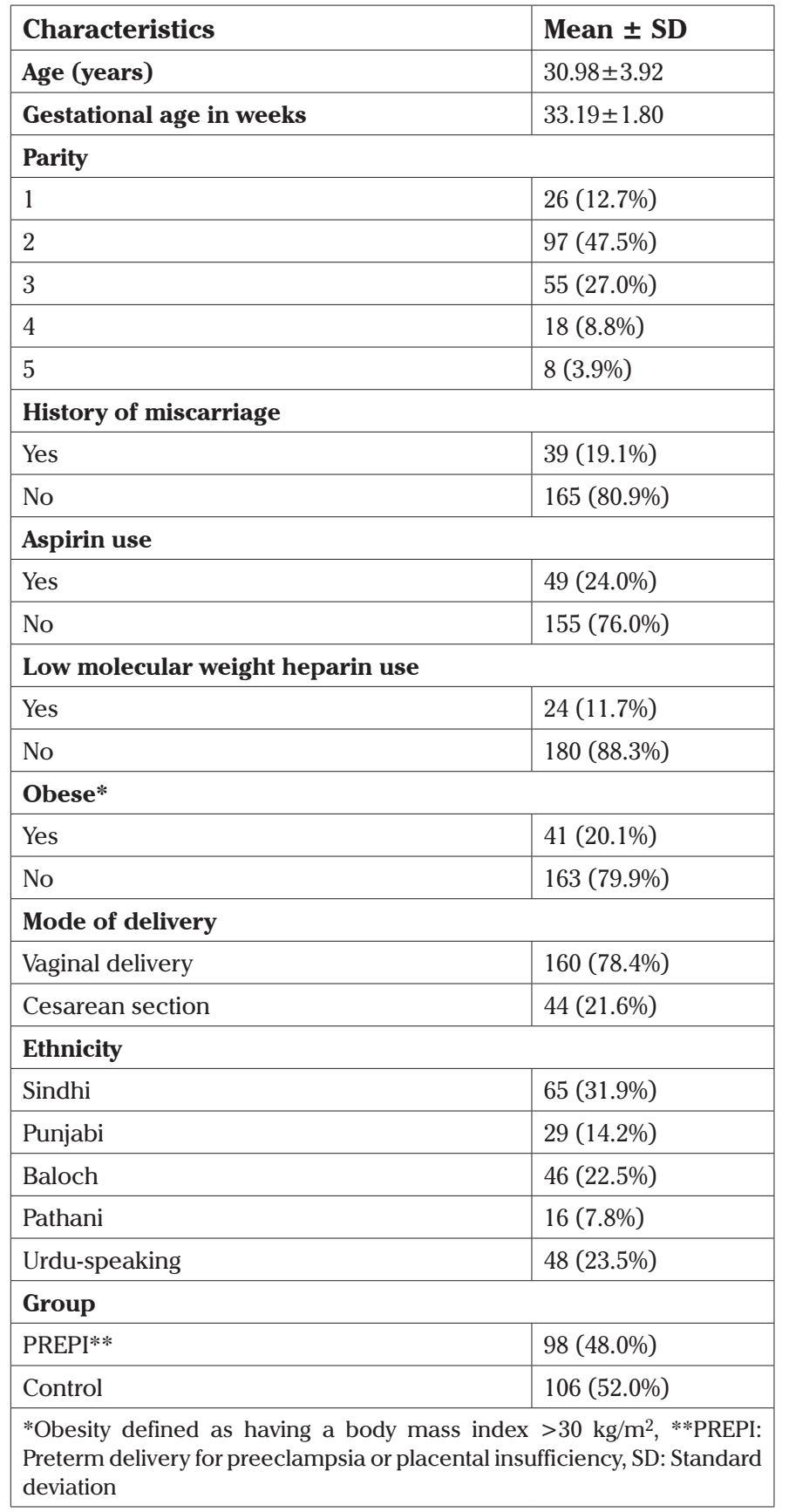


LAC was the most common antibody found in women who deliver preterm with PREPI.

\section{Strengths and limitations}

The strength of this study is its prospective design and application of Sapporo's criteria of repeat testing and antibody level cut-offs were adhered to, to confirm APS in women with PREPI. International, standard ACOG criteria were used for diagnosing preeclampsia with severe features to guide screening in this group. Our study would aid in strengthening evidence in cases of PREPI for postnatal workup that includes testing for APLA in this high risk group. Follow up of all patients was maintained to ensure the prevalence was not underestimated.

The effect of treatment (aspirin and low molecular weight heparin) was not assessed in these diagnosed cases so it is not possible to comment on the effect of instituting treatment. This could be assessed by future larger trials.

Table 2. Comparison of cases and controls

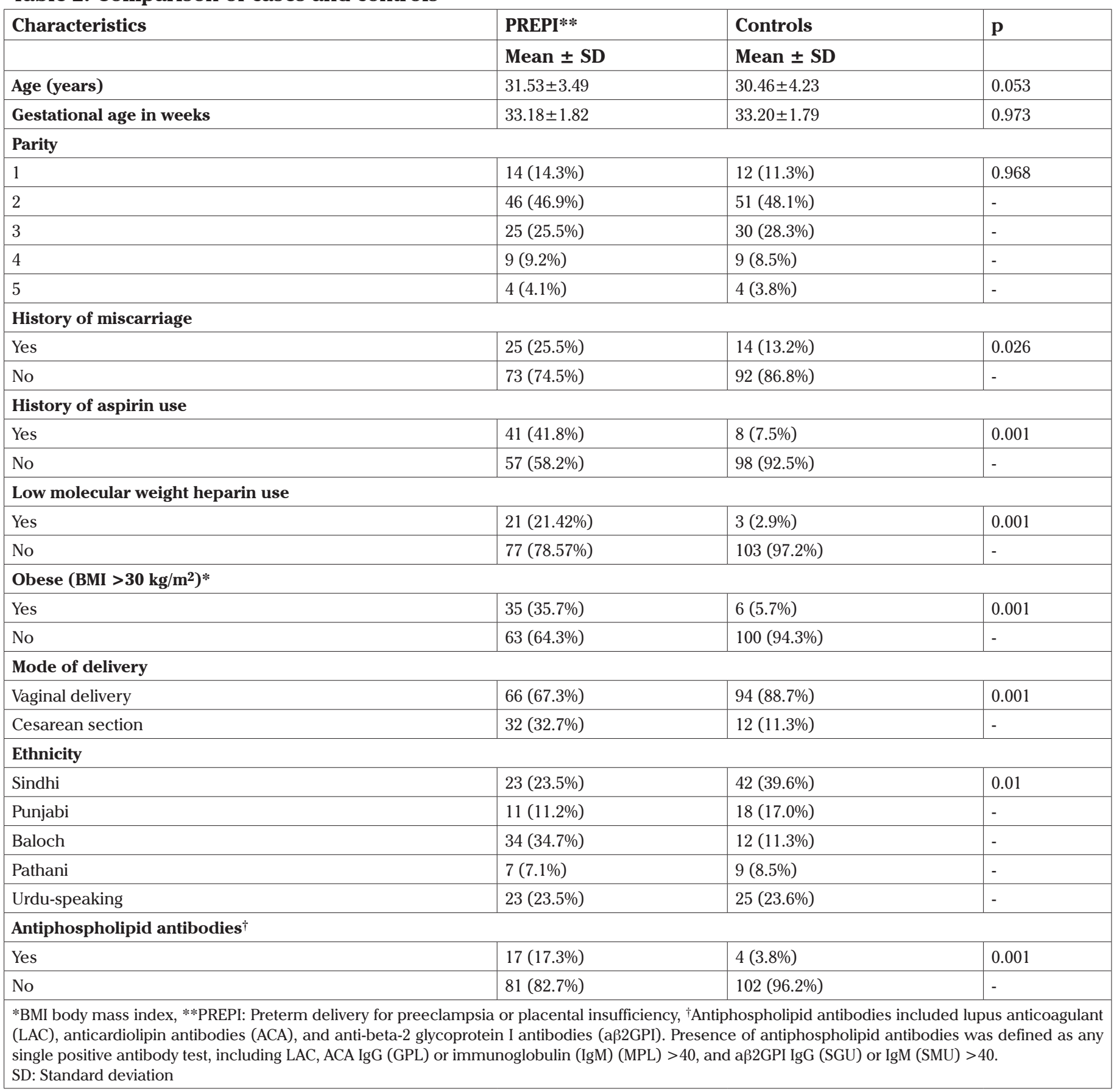




\section{Interpretation}

The association of APLA with preeclampsia has been investigated extensively and two meta-analyses have assessed this association. In the first meta-analysis that included 12 studies, criteria for preeclampsia were classified in only six of the included studies (15). Similarly in the second metaanalysis, the association between LAC and preeclampsia was found by five included studies but preterm delivery was not included as an outcome measure in any of the 28 studies that were analysed for the systematic review (16). The only study that investigated preterm delivery suffered due to a lack of follow-up and repeat testing for antibodies, which was denoted as a weakness of the study and potential area for future work (10). Our study rectifies this omission as follow-up was maintained in all cases and repeat testing was ensured. In a recent study the authors maintained follow up in 52.6\% of cases and but noted that the study was limited because of the criteria implemented for preterm delivery and APS (10). The authors included women delivering up to 36 weeks whereas the Saporo criterion gives 34 weeks as the threshold. We included all women who delivered before 34 weeks and therefore ensured that this weakness was also rectified and the evidence base has been strengthened concerning the utility of APLA screening in preeclampsia.

Insufficient evidence in such cases has continued to hinder implementation of testing for APLA in cases of severe preeclampsia (17). The likely benefit of instituting treatment in these cases would more appropriately assess the usefulness of investigation. This is an area for future research. We suggest further studies that look into the role of such intervention in appropriately diagnosed cases.

Table 3. Antibodies in both control and cases

\begin{tabular}{|c|c|c|}
\hline \multirow[t]{2}{*}{ Antibodies* } & \multicolumn{2}{|l|}{ Group } \\
\hline & $\begin{array}{l}\text { PREPI } \\
(n=17)\end{array}$ & $\begin{array}{l}\text { Control } \\
(n=4)\end{array}$ \\
\hline \multicolumn{3}{|c|}{ Lupus anticoagulant } \\
\hline Yes & $14(82.4 \%)$ & $3(75.0 \%)$ \\
\hline No & $3(17.6 \%)$ & $1(25.0 \%)$ \\
\hline \multicolumn{3}{|c|}{ Anticardiolipin antibodies (IgG/IgM)** } \\
\hline Yes & $8(47.1 \%)$ & $2(50.0 \%)$ \\
\hline No & $9(52.9 \%)$ & $2(50.0 \%)$ \\
\hline \multicolumn{3}{|c|}{ Anti- $\beta 2$-glycoprotein I antibodies (IgG/IgM)** } \\
\hline Yes & $3(17.6 \%)$ & $1(25.0 \%)$ \\
\hline No & $14(82.4 \%)$ & $3(75.0 \%)$ \\
\hline \multicolumn{3}{|c|}{$\begin{array}{l}\text { *Any antibody should be demonstrable on at least two occasions } \\
\text { separated by } 12 \text { weeks, **Both immunoglobulin G (IgG) and IgM } \\
\text { were done, due to the small number in control group, the results are } \\
\text { not shown individually, PREPI: Preterm delivery for preeclampsia or } \\
\text { placental insufficiency }\end{array}$} \\
\hline
\end{tabular}

The proportion of women in our population that tested positive for APLA was higher than anticipated. In a study from Utah, 1 in every 9 women, just over $11 \%$, who delivered preterm due to a severe form of preeclampsia had APLA, whereas in our study the proportion was much higher 17.3\% (approaching 1 in 5). If repeat testing for confirmation of APS had been performed in the Utah study, a similar prevalence of APLA may have been identified (10). However, on the basis of their findings, these authors claimed that 1 in 9 is sufficiently high to propose screening. We would agree with their statement and add that if the true incidence is closer to 1 in 5 , all women who deliver before 34 weeks due to PREPI must undergo screening for APLA to confirm APS. Our study findings also suggest that Pakistani-Asians are a high risk group for this screening.

In our study the cases were more likely to have LAC $(n=14$, $82.4 \%$ vs. $n=3,75 \%$ ) whereas controls were more likely to have ACL $(n=2,50 \%$ vs. $n=8,47 \%)$. Hence LAC is more specific for PREPI. This finding was similar to other reports (18).

APS diagnosis requires one lab and one clinical criterion to be positive. Although PREPI is a clinical criterion and thus all women who deliver early because of PREPI already meet the clinical criteria, few women who deliver preterm due to PREPI are ever screened for APLA, and thus the presence or absence of the lab criterion is unknown in most cases (11). Women with APS need special management to prevent further adverse pregnancy outcomes, which cannot be instituted if this opportunity is missed. We counseled and screened all women with PREPI. Our study shows a high enough prevalence to warrant screening in this group.

There is no clear consensus on screening APS in the obstetric population. The Royal College of Obstetricians and Gynaecologists (19) and the Society of Obstetricians and Gynaecologists of Canada (20) do not provide any guidance on APS screening for women with early-onset, severe hypertensive disease of pregnancy whereas the Royal Australian and New Zealand College of Obstetricians and Gynecologists recommends APS screening in these cases (21). In contrast, ACOG recommends against routine screening in the population (22).

Another argument against routine screening is due to lack of data that supports improved outcomes in women with APS. However a recent study showed improved outcomes with targeted therapy (23). We will thus continue to miss an excellent opportunity if we do not provide targeted screening. Our study provides evidence that screening in this population can be fruitful and may lead to better outcomes in future pregnancies.

\section{Conclusion}

Our study confirms a high prevalence of APLA in women with PREPI. Testing in this high-risk group can lead to better 
fetomaternal and neonatal outcomes. An opportunity to screen these women should not be missed, so that proper counselling can be given and future pregnancies can be managed in an appropriate and timely manner. Screening is insufficient in our region and policy should be implemented to at least target women with PREPI. Given the difference in prevalence reported in this and the American study, it is suggested that the prevalence of APLA in women delivering at or before 34 weeks gestational age due to PREPI should be determined in other populations.

Ethics Committee Approval: The study was approved by the Institutional Ethics Committee of Aziz Medical Center (approval number: IEC/AZIZ/334).

Informed Consent: All participants provided informed consent.

Peer-review: Externally peer-reviewed.

Author Contributions: Surgical and Medical Practices: R.I., S.H.A., Sa.H., S.H.; Concept - R.I., S.H.A., Sa.H., S.H.; Design - R.I., S.H.A., Sa.H., S.H.; Data Collection or Processing - R.I., S.H.A., Sa.H., S.H.; Analysis or Interpretation - R.I., S.H.A., Sa.H., S.H.; Literature Search - Sa.H.; Writing - R.I., S.H.A., Sa.H., S.H.

Conflict of Interest: No conflict of interest is declared by the authors.

Financial Disclosure: The authors declared that this study received no financial support.

\section{References}

1. Park SH, Jang S, Park CJ, Chi HS. Clinical Application of Revised Laboratory Classification Criteria for Antiphospholipid Antibody Syndrome: Is the follow-up interval of 12 weeks instead of 6 weeks significantly useful? Biomed Res Int 2016; (7): 1-6.

2. Nilsen RM, Vik ES, Rasmussen SA, Small R, Moster D, Schytt E, et al. Preeclampsia by maternal reasons for immigration: a populationbased study. BMC Pregnancy Childbirth 2018; 18: 423.

3. Fantasia I, Andrade W, Syngelaki A, Akolekar R, Nicolaides KH Impaired placental perfusion and major fetal cardiac defects. Ultrasound Obstet Gynecol 2019; 53: 68-72.

4. Antovic A, Sennström M, Bremme K, Svenungsson E. Obstetric antiphospholipid syndrome. Lupus Sci Med. 2018; 5: e000197.

5. Yamada H, Atsumi T, Amengual O, Koike T, Furuta I, Ohta K, et al. Anti-beta2 glycoprotein-I antibody increases the risk of pregnancyinduced hypertension: a case-controlled study. J Reprod Immunol 2010; 84: 95-9.

6. Chauleur C, Galanaud JP, Alonso S, Cochery-Nouvellon E, Balducchi JP, Marès $\mathrm{P}$, et al. Observational study of pregnant women with a previous spontaneous abortion before the 10th gestation week with and without antiphospholipid antibodies. J Thromb Haemost 2010; 8: 699-706.

7. Mayer-Pickel K, Eberhard K, Lang U, Cervar-Zivkovic M. Pregnancy Outcome in Women with Obstetric and Thrombotic
Antiphospholipid Syndrome-A Retrospective Analysis and a Review of Additional Treatment in Pregnancy. Clin Rev Allergy Immunol 2017; 53: 54-67.

8. Mello G, Parretti E, Marozio L, Pizzi C, Lojacono A, Frusca T, et al. Thrombophilia is significantly associated with severe preeclampsia: results of a large-scale, case-controlled study. Hypertension 2005; 46: $1270-4$.

9. de Jesus GR, Agmon-Levin N, Andrade CA, Andreoli L, Chighizola CB, Porter TF, et al. 14th International Congress on Antiphospholipid Antibodies Task Force report on obstetric antiphospholipid syndrome. Autoimmun Rev 2014; 13: 795-813.

10. Gibbins KJ, Tebo AE, Nielsen SK, Branch DW. Antiphospholipid antibodies in women with severe preeclampsia and placental insufficiency: a case-control study. Lupus 2018; 27: 1903-10.

11. Sobhani NC, Shulman R, Tran EE, Gonzalez JM. Early Onset Severe Hypertensive Disease in Pregnancy and Screening for Antiphospholipid Syndrome. AJP Rep 2020; 10: e32-e6.

12. Roberts JM, August PA, Bakris G, Barton JR, Bernstein IM, Druzin $\mathrm{M}$, et al. Hypertension in pregnancy: executive summary. Obstet Gynecol 2013; 122: 1122-31.

13. Miyakis S, Lockshin MD, Atsumi T, Branch DW, Brey RL, Cervera $\mathrm{R}$, et al. International consensus statement on an update of the classification criteria for definite antiphospholipid syndrome (APS). J Thromb Haemost 2006; 4: 295-306.

14. Pengo V, Tripodi A, Reber G, Rand JH, Ortel TL, Galli M, et al; Subcommittee on Lupus Anticoagulant/Antiphospholipid Antibody of the Scientific and Standardisation Committee of the International Society on Thrombosis and Haemostasis. Update of the guidelines for lupus anticoagulant detection. Subcommittee on Lupus Anticoagulant/Antiphospholipid Antibody of the Scientific and Standardisation Committee of the International Society on Thrombosis and Haemostasis. J Thromb Haemost 2009; 7: 1737-40.

15. do Prado AD, Piovesan DM, Staub HL, Horta BL. Association of anticardiolipin antibodies with preeclampsia: a systematic review and meta-analysis. Obstet Gynecol 2010; 116: 1433-43.

16. Abou-Nassar K, Carrier M, Ramsay T, Rodger MA. The association between antiphospholipid antibodies and placenta mediated complications: a systematic review and meta-analysis. Thromb Res 2011; 128: 77-85.

17. de Jesus GR, Agmon-Levin N, Andrade CA, Andreoli L, Chighizola $\mathrm{CB}$, Porter TF, et al. 14th International Congress on Antiphospholipid Antibodies Task Force report on obstetric antiphospholipid syndrome. Autoimmun Rev 2014; 13: 795-813.

18. Lockshin MD, Kim M, Laskin CA, Guerra M, Branch DW, Merrill J, et al. Prediction of adverse pregnancy outcome by the presence of lupus anticoagulant, but not anticardiolipin antibody, in patients with antiphospholipid antibodies. Arthritis Rheum 2012; 64: 2311-8.

19. Royal College of Obstetricians and Gynaecologists. Reducing the Risk of Venous Thromboembolism during Pregnancy and the Puerperium. London: Green-Top Guideline Number 37a; 2015.

20. Magee LA, Pels A, Helewa M, Rey E, von Dadelszen P; Canadian Hypertensive Disorders of Pregnancy Working Group. Diagnosis, evaluation, and management of the hypertensive disorders of pregnancy: executive summary. J Obstet Gynaecol Can 2014; 36: 416-41.

21. Lowe SA, Bowyer L, Lust K, McMahon LP, Morton M, North RA, et al. SOMANZ guidelines for the management of hypertensive disorders of pregnancy 2014. Aust N Z J Obstet Gynaecol 2015; 55: e1-e29.

22. Committee on Practice Bulletins-Obstetrics, American College of Obstetricians and Gynecologists. Practice Bulletin No. 132: antiphospholipid syndrome. Obstet Gynecol 2012; 120: 1514-21.

23. Hoxha A, Favaro M, Calligaro A, Del Ross T, Ruffatti AT, Infantolino C, et al. Upgrading Upgrading Therapy Strategy Improves Pregnancy Outcome in Antiphospholipid Syndrome: A Cohort Management Study. Thromb Haemost 2020; 120: 36-43. 